Phys. Rev. Lett. 100, 184501 (2008); E-print arXiv:0710.3359

\title{
Generation of Magnetic Field by Combined Action of Turbulence and Shear
}

\author{
T. A. Yousef, ${ }^{1}$ T. Heinemann, ${ }^{1}$ A. A. Schekochihin, ${ }^{2}$ N. Kleeorin,${ }^{3}$ \\ I. Rogachevskii, ${ }^{3}$ A. B. Iskakov, ${ }^{4,2}, 5$ S. C. Cowley, ${ }^{4,2}$ and J. C. McWilliams ${ }^{6}$ \\ ${ }^{1}$ DAMTP, University of Cambridge, Cambridge CB3 0WA, United Kingdom \\ ${ }^{2}$ Plasma Physics Group, Blackett Laboratory, Imperial College, London SW7 2AZ, United Kingdom \\ ${ }^{3}$ Department of Mechanical Engineering, The Ben-Gurion University of the Negev, P. O. Box 653, Beer-Sheva 84105, Israel \\ ${ }^{4}$ Department of Physics and Astronomy, UCLA, Los Angeles, California 90095-1547, USA \\ ${ }^{5}$ Wolfgang Pauli Institute, University of Vienna, Nordbergstraße 15, A-1090 Vienna, Austria \\ ${ }^{6}$ Department of Atmospheric Sciences, UCLA, Los Angeles, California 90095-1565, USA
}

(Dated: October 31, 2018)

\begin{abstract}
The feasibility of a mean-field dynamo in nonhelical turbulence with superimposed linear shear is studied numerically in elongated shearing boxes. Exponential growth of magnetic field at scales much larger than the outer scale of the turbulence is found. The charateristic scale of the field is $l_{\bar{B}} \propto S^{-1 / 2}$ and growth rate is $\gamma \propto S$, where $S$ is the shearing rate. This newly discovered shear dynamo effect potentially represents a very generic mechanism for generating large-scale magnetic fields in a broad class of astrophysical systems with spatially coherent mean flows.
\end{abstract}

PACS numbers: 47.65.Md, 47.27.W-, 95.30.Qd, 98.62.En

Introduction. Understanding the origin of cosmic magnetism is one of the fundamental theoretical challenges in astrophysics. The turbulent motions of the plasmas that make up most astrophysical objects are believed to be responsible for the generation of the magnetic field. In particular, a generic property of the turbulence of conducting fluid is to amplify exponentially magnetic fluctuations at the turbulence scales or smaller via the fluctuation dynamo effect [1, 2, 3]. A distinct problem is to explain the observed presence in most astrophysical bodies of magnetic fields spatially coherent at scales larger than the outer scale of the turbulence (mean fields). Nonhelical homogeneous isotropic turbulence on its own cannot give rise to a mean field. What are then the large-scale properties that must be present in a turbulent system for such a field to be generated? Mean-field dynamo theories [4] have identified a number of amplification mechanisms. We know, e.g., that nonzero net helicity (often combined with rotation in real systems) is sufficient to produce mean fields, but is it necessary?

Perhaps the most common large-scale feature is a mean velocity shear. It is present, e.g., in stellar interiors [5], accretion disks [6], galaxies (in particular, irregular ones [7]), and liquid-metal laboratory dynamos [8], all of which host both large-scale (mean) and small-scale (fluctuating) magnetic fields. A number of theories have proposed that a mere combination of turbulence and shear could give rise to a mean-field dynamo: e.g., the shear-current effect [9], the stochastic $\alpha$ effect [10], shear amplification of small-scale-dynamo-generated field [11], negativediffusivity-type theories [12]. Ref. [9], which used the $\tau$-approximation closure, provoked a particular debate because it seemed to contradict the rigorous mean field theory based on the second-order correlation approximation (SOCA), which ruled out the shear dynamo [13].
However, the SOCA is only valid in the limit either of low hydrodynamic and magnetic Reynolds numbers, $\mathrm{Re}, \mathrm{Rm} \ll 1$, or short velocity correlation times [4]. The real turbulent systems are in neither of these limits, and the hope that some of the results qualitatively carry over has had to be backed up by numerical evidence [14 and by intuitive physical field-amplification scenarios [15]. In the absence of a compelling physical argument for or against the shear dynamo or of a rigorous method for proceeding analytically, a numerical experiment is overdue. Here we report the first such numerical experiment, which supports the existence of the shear dynamo.

Numerical Set Up. We consider the incompressible magnetohydrodynamics (MHD) with a background linear shear flow $\mathbf{U}=-S x \hat{\mathbf{y}}$ and a white-noise nonhelical random homogeneous isotropic body force $\mathbf{f}$ :

$$
\begin{aligned}
\frac{d \mathbf{u}}{d t} & =u_{x} S \hat{\mathbf{y}}-\frac{\nabla p}{\rho}+\frac{\mathbf{B} \cdot \nabla \mathbf{B}}{4 \pi \rho}+\nu \nabla^{2} \mathbf{u}+\mathbf{f}, \\
\frac{d \mathbf{B}}{d t} & =-B_{x} S \hat{\mathbf{y}}+\mathbf{B} \cdot \nabla \mathbf{u}+\eta \nabla^{2} \mathbf{B},
\end{aligned}
$$

where $\mathbf{u}$ and $\mathbf{B}$ are the velocity and magnetic fields, $d / d t=\partial_{t}-S x \partial_{y}+\mathbf{u} \cdot \boldsymbol{\nabla}$, the density $\rho=1$, and the pressure $p$ is determined by the incompressibility condition $\boldsymbol{\nabla} \cdot \mathbf{u}=0$. These equations are solved with shear-periodic boundary conditions by a Lagrangian spectral method [16]. When the imposed shear $S$ is weak compared to the turnover rate of the turbulent motions, the growth of the mean (large-scale) field can only be detected if the size of the computational domain is much larger than the turbulence scale $l_{0}$. In general, this, together with the necessity to run the simulations for very long times, requires unaffordable amounts of computing power. We circumvent this problem by using computational boxes with large aspect ratios, $L_{x} \times L_{y} \times L_{z}$, where $L_{z} \gg L_{x}=L_{y}$. The units of length and time are fixed by setting $L_{x}=L_{y}=1$ 
TABLE I: Index of runs

\begin{tabular}{llllrr}
\hline \hline$S$ & $L_{z}$ & Resolution & $\gamma$ & $l_{\bar{B}}$ & $\bar{B}_{y} / \bar{B}_{x}{ }^{a}$ \\
\hline 2 & 8 & $32^{2} \times 256$ & 0.0161 & 3.7 & 6.54 \\
2 & 16 & $32^{2} \times 512$ & 0.021 & 3.8 & 6.49 \\
\hline 1 & 8 & $32^{2} \times 256$ & 0.0030 & 4.6 & 6.38 \\
1 & 16 & $32^{2} \times 512$ & 0.0124 & 5.4 & 6.50 \\
1 & 32 & $32^{2} \times 1024$ & 0.0092 & 5.2 & 6.43 \\
1 & 64 & $32^{2} \times 2048$ & 0.0121 & 5.1 & 6.35 \\
\hline 0.5 & 16 & $32^{2} \times 512$ & 0.0040 & 6.8 & 6.34 \\
0.5 & 32 & $32^{2} \times 1024$ & 0.0058 & 7.1 & 6.31 \\
0.5 & 64 & $32^{2} \times 2048$ & 0.0055 & 7.3 & 6.32 \\
\hline 0.25 & 64 & $32^{2} \times 2048$ & 0.0025 & 9.7 & 6.07 \\
0.25 & 128 & $32^{2} \times 4096$ & 0.0025 & 9.9 & 6.06 \\
\hline 0.125 & 64 & $32^{2} \times 2048$ & 0.00094 & 13.1 & 6.01 \\
0.125 & 128 & $32^{2} \times 4096$ & 0.00092 & 13.5 & 6.02 \\
\hline \hline
\end{tabular}

${ }^{a}$ This is the time average of $\left[\int d z \bar{B}_{y}^{2}(z) / \int d z \bar{B}_{x}^{2}(z)\right]^{1 / 2}$.

and the mean forcing power $\epsilon=\langle\mathbf{u} \cdot \mathbf{f}\rangle=1$ (this can be controlled because the forcing is white-noise). The average forcing scale is $l_{0}=1 / 3$, i.e., the energy is injected randomly in the wave-number shell centered at $k_{0} / 2 \pi=3$. The resulting root-mean-square velocity field is $u_{\mathrm{rms}} \equiv\left\langle u^{2}\right\rangle^{1 / 2} \simeq 1 \pm 0.3$, so the typical turnover rate of the turbulent motions is $u_{\mathrm{rms}} / l_{0} \sim 3$. We study five values of the shear $S=2,1,1 / 2,1 / 4,1 / 8<u_{\mathrm{rms}} / l_{0}$ (weak shear is used in order for the mean and fluctuating fields to be clearly distinguishable from each other via small-scale averaging; see Eq. (3) below). The viscosity and magnetic diffusivity are $\nu=\eta=10^{-2}$, so $\mathrm{Rm}=\mathrm{Re} \equiv u_{\mathrm{rms}} / k_{0} \nu \sim 5$. The resolution requirements are consequently not large: it suffices to have $32 \times 32$ collocation points in the $(x, y)$ plane. In the $z$ direction, we use resolutions between 256 and 4096 collocation points for $L_{z}=8, \ldots, 128$, depending on $S$ (Tab. I).

Strictly speaking, we cannot speak about turbulence with such low Re. However, a developed inertial range is not important for mean field dynamos: it is sufficient that a stochastic velocity field with $\mathrm{Re} \gtrsim 1$ is present 21 . In our simulations, $\mathrm{Rm}$ is subcritical with respect to the fluctuation dynamo [2, 3], so any field growth we detect is due purely to a mean-field dynamo. Note, however, that since $\mathrm{Rm}>1$, turbulent tangling of the mean field generates small-scale magnetic fluctuations whose energy is in general not smaller than that of the mean field [3].

Results. All runs are initialized with a random, zeromean, dynamically weak $\left(\left\langle B^{2}\right\rangle=10^{-20}\right)$ magnetic field. The field grows exponentially with time at all values of $S$ studied, provided the computational box is sufficiently long. For each $S$, we consider the growth rate $\gamma$ of $B_{\text {rms }} \equiv\left\langle B^{2}\right\rangle^{1 / 2}$ to be converged if it stays approximately the same when $L_{z}$ is doubled (Fig. 11). That we are able to find such values means that $\gamma$ is asymptotically inde-
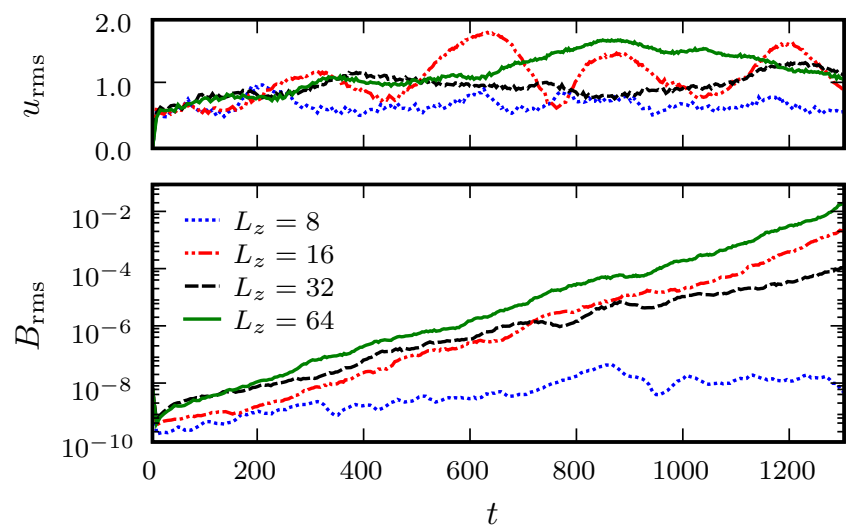

FIG. 1: Evolution of $u_{\mathrm{rms}}$ (upper panel) and $B_{\mathrm{rms}}$ (lower panel) for $S=1$ and four values $L_{z}=8,16,32,64$.

pendent of $L_{z}$ (the dependence on $L_{x}$ and $L_{y}$ should also be studied but that is currently too expensive computationally). The field eventually grows to a dynamically strong saturated level. Here we concentrate on the kinematic (weak-field) regime and leave the properties of the saturated state to a future study.

Fig. 2 shows that, in the range of shears studied, the growth rate of $B_{\mathrm{rms}}$ appears to increase linearly with $S$, $\gamma \propto S$. This is a somewhat unexpected result from the theoretical point of view because the shear-current effect [9], as well as most other mean-field theories quoted above predict $\gamma \propto S^{2}$ for the fastest-growing mode. We cannot, however, exclude the possibility that the $S^{2}$ scaling may be asymptotically recovered at even smaller $S$.

That the growing field is large-scale is obvious already from the visualization of the field: the large-scale $z$ dependent modulation is evident against the turbulencescale structure (Fig. 3). We isolate this large-scale dependence on $z$ by low-pass filtering in Fourier space:

$$
\overline{\mathbf{B}}(z)=\sum_{\left|k_{z} / 2 \pi\right|<1} \mathbf{B}\left(k_{x}=0, k_{y}=0, k_{z}\right) e^{i k_{z} z} .
$$

Note that since $\nabla \cdot \overline{\mathbf{B}}=0, \bar{B}_{z}=0$. This procedure averages out the small-scale structure and brings out the growing large-scale field in a clear way (Fig. 3). In all cases, the root-mean-square values of $\bar{B}_{x}$ and $\bar{B}_{y}$ grow exponentially with the same rate $\gamma$ as $B_{\text {rms. }}$. We find $\left|\bar{B}_{y}\right|>\left|\bar{B}_{x}\right|$, which is expected because the shear systematically converts $B_{x}$ into $B_{y}$ [Eq. (2)]. The ratio $\left|\bar{B}_{y} / \bar{B}_{x}\right| \sim S / \gamma$ is approximately constant in time and its average is independent of $S$ (Tab. I), which is consistent with $\gamma \propto S$ established above.

Examining Fig. 3, we see that the magnetic field grows in large random patches. In time, they move around (in $z$ ) and change shape in an apparently random fashion. This means that the mean field is not strictly speaking an "eigenmode" with a spatial profile constant in time, although we found the correlation time scale of its evolution to be much longer than either the turnover time $\left(l_{0} / u_{\mathrm{rms}}\right)$ or the shear time $\left(S^{-1}\right)$. In order to describe 


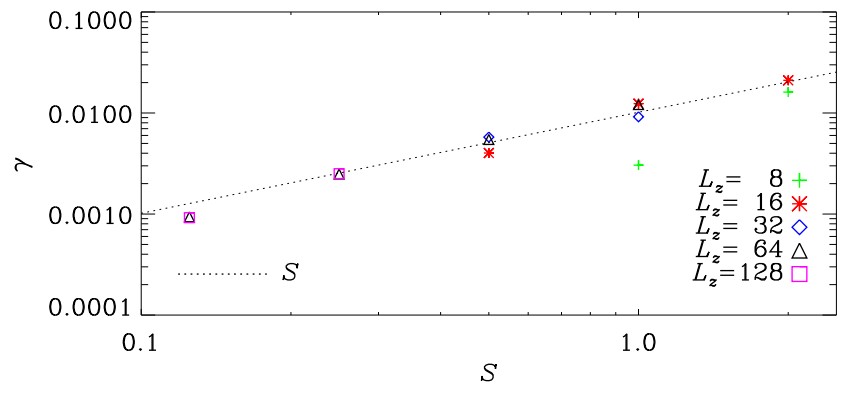

FIG. 2: Growth rates $\gamma$ of $B_{\text {rms }}$ for all runs (Tab. 【). The dotted line shows the slope corresponding to $\gamma \propto S$.

the spatial structure of the mean field in a systematic way, we define the time-averaged characteristic scale $l_{\bar{B}}$ :

$$
\frac{1}{l_{\bar{B}}}=\frac{1}{t_{2}-t_{1}} \int_{t_{1}}^{t_{2}} d t\left[\frac{\int d z\left(\partial \bar{B}_{y} / \partial z\right)^{2}}{\int d z \bar{B}_{y}^{2}}\right]^{1 / 2} .
$$

Here and in all other cases, the time average is taken over the exponential-growth (kinematic) period $\left(t_{1}, t_{2}\right)$ of the field evolution. The derivatives are calculated in Fourier space. The values of $l_{\bar{B}}$ are given in Tab. I and plotted vs. $S$ in Fig. 目 As the shear is decreased, $l_{\bar{B}}$ increases and is well matched by the scaling $l_{\bar{B}} \propto S^{-1 / 2}$.

This scaling is again at odds with the mean-field-theory prediction $l_{\bar{B}} \propto S^{-1}[9]$, but the following simple argument shows that it is consistent with $\gamma \propto S$ and suggests the possible form that a mean-field theory of the dynamo reported here may take. Let us conjecture the mean-field equations in the following (standard) model form

$$
\begin{aligned}
& \partial_{t} \bar{B}_{x}=-\eta_{T} k_{z}^{2} \bar{B}_{x}-A \bar{B}_{y}, \\
& \partial_{t} \bar{B}_{y}=-\eta_{T} k_{z}^{2} \bar{B}_{y}-S \bar{B}_{x},
\end{aligned}
$$

where $\eta_{T} \sim u_{\mathrm{rms}} l_{0}$ is the turbulent diffusivity and $A$ is some operator that closes the dynamo loop. (the challenge of mean-field theory is to find $A$ ). The growth
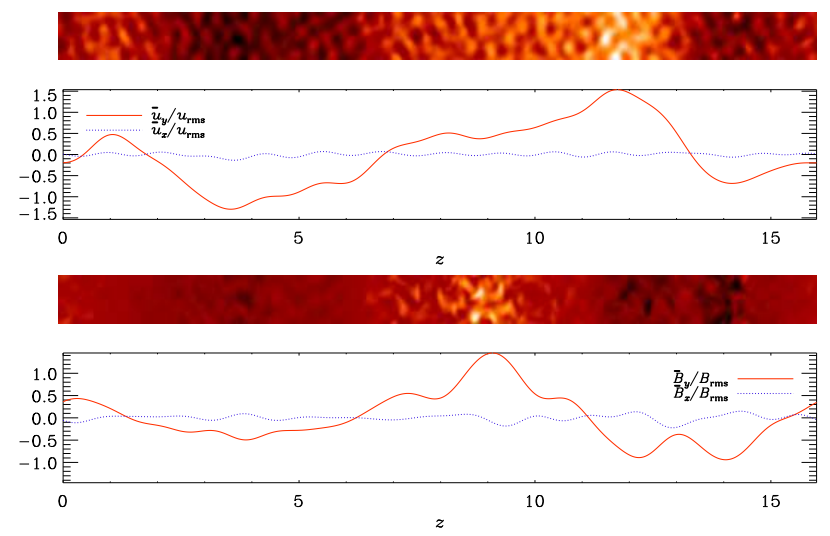

FIG. 3: Snapshots of $u_{y}$ (upper panel) and $B_{y}$ (lower panel) taken in an $(y, z)$ cross-section of the $L_{z}=16$ run for $S=1$ (the forcing scale is $l_{0}=1 / 3$ ). Underneath the snapshots are plots of $\bar{u}_{y}(z), \bar{u}_{x}(z)$ (upper panel) and $\bar{B}_{y}(z), \bar{B}_{x}(z)$ (lower panel). Here $\overline{\mathbf{u}}(z)$ is defined similarly to $\overline{\mathbf{B}}(z)$ [Eq. (3)].

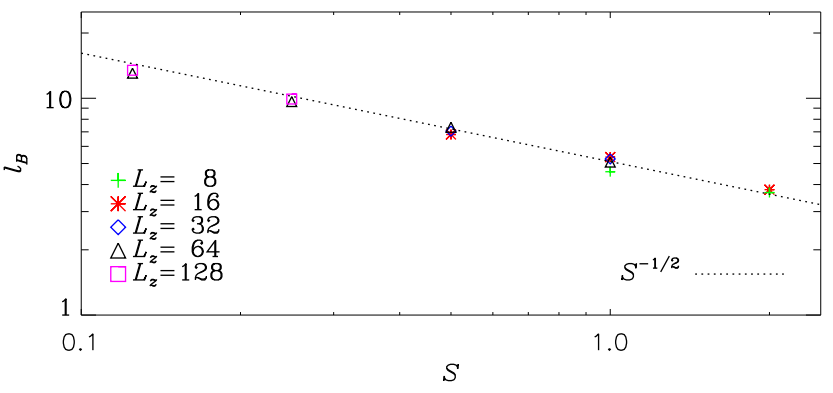

FIG. 4: The characteristic scale of the magnetic field [Eq. (4)] for all runs. The dotted line showes the slope $S^{-1 / 2}$.

rate is $\gamma=\sqrt{S A}-\eta_{T} k_{z}^{2}$. For the fastest-growing mode, the two terms in this expression are comparable, so, if $k_{z} \sim l_{\bar{B}}^{-1} \sim l_{0}^{-1}\left(S l_{0} / u_{\mathrm{rms}}\right)^{1 / 2}$, then $A \sim S$ and $\gamma \sim S$ (in contrast, some mean-field theories predict $A \sim S l_{0}^{2} k_{z}^{2}$ [9, 10], so $k_{z} \propto S$ and $\gamma \propto S^{2}$ ).

Finally, in Fig. 罒, we show the spectrum of magnetic energy during the growth stage. It is strongly peaked at large scales $\left(k_{z} l_{0} \ll 1\right)$, but also shows that the mean field is tangled by the turbulence to produce a significant amount of magnetic energy at the turbulence scales. The presence of this tangled component is likely to be essential in the shear dynamo effect in that the small-scale field could be continuously resupplying the field $B_{x}$ for amplification by shear [11]. The key to a successful theory of the shear dynamo may be to find the way in which the symmetry is broken to make this resupply systematically favorable to the exponential growth of the mean field (as, e.g., implicitly attempted in Ref. [9]).

Effect of Shear on Velocity Field. It has been suggested that a mechanism similar to the shear dynamo may also produce large-scale velocity structures ("vorticity dynamo" [17]). The velocity does indeed develop large fluctuations that are energetically comparable to

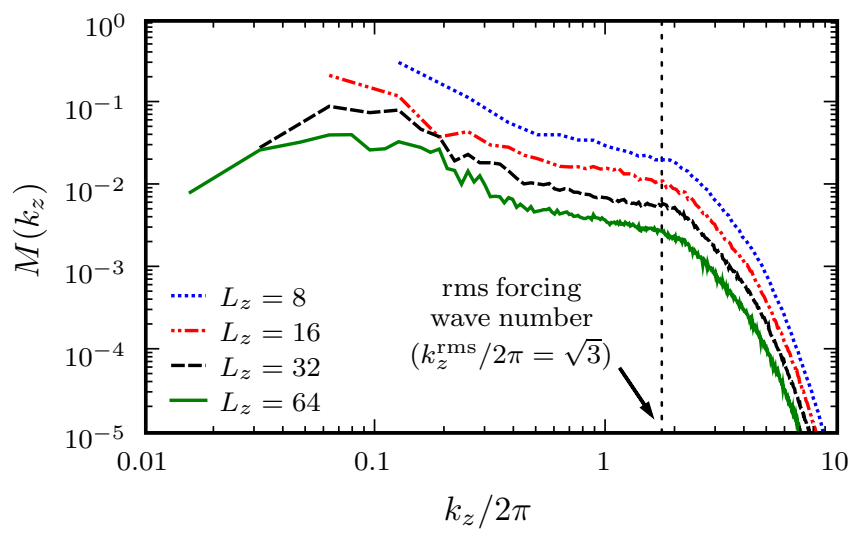

FIG. 5: Normalized one-dimensional spectra of the magnetic energy, $M\left(k_{z}\right)=\sum_{k_{x}, k_{y}}\left|\mathbf{B}\left(k_{x}, k_{y}, k_{z}\right)\right|^{2} /\left\langle B^{2}\right\rangle$ (time averaged over the growth stage) for $S=1$ and $L_{z}=8,16,32,64$. The four graphs demonstrate that, as $L_{z}$ is increased, a large-scale spatial structure independent of the box length emerges. 
the small-scale turbulence, last for long times (Fig. 1) and are spatially coherent on scales similar to those of the magnetic field (Fig. 3). The large-scale structure forms mainly in $u_{y}$ (corresponding to large-scale vorticity, $\left.\bar{\omega}_{x}=-\partial \bar{u}_{y} / \partial z\right)$. As the forcing always seeds some largescale vorticity that is not infinitesimally small, the "vorticity dynamo" always operates in the nonlinear regime. Its detailed study is outside the scope of this Letter.

The growth of the magnetic field does not seem to be strongly correlated with the evolution of the sheargenerated large-scale velocity structures (compare, e.g., the time evolution of $u_{\mathrm{rms}}$ and $B_{\mathrm{rms}}$ in Fig. 1).

It is well known that the presence of shear can lead to nonlinear destabilization of finite velocity fluctuations and formation of shear-driven turbulence whose outer scale is the scale of the shear (in simulations with a linear shear, the box scale). This does indeed happen in our simulations when $S$ is too strong or the box is too long. The quantitative signature of this regime is that the power input from the shear in Eq. (11), $\left\langle u_{x} u_{y}\right\rangle S$, exceeds the forcing power $\epsilon=\langle\mathbf{u} \cdot \mathbf{f}\rangle$. We avoid this regime to isolate the mean-field generation effect, which requires a scale separation between the turbulence and the mean field. In all runs reported here, $\left|\left\langle u_{x} u_{y}\right\rangle S\right| \ll \epsilon$. We note that the large upward fluctuations of $u_{\mathrm{rms}}$ [Fig. [1] are not accompanied by a significant change in $\left\langle u_{x} u_{y}\right\rangle S$, so the large-scale velocity structures appear to feed on the forcing power, not on the power extracted from the shear.

Discussion. We have found that a large-scale magnetic field grows exponentially in long sheared boxes with forced small-scale nonhelical turbulence. In the parameter range we have studied, the growth rate appears to scale as $\gamma \propto S$, the spatial scale of the field $l_{\bar{B}} \propto S^{-1 / 2}$ and $\bar{B}_{y} / \bar{B}_{x} \simeq$ const $>1$. These properties do not seem to fit any of the existing theories. Our results do, however, lend credence to the concept of a shear dynamo and thus provide motivation for further theoretical effort.

To our knowledge, this is the first demonstration of the shear dynamo effect in a dedicated numerical experiment. In an earlier unpublished study we obtained similar results using PENCIL code (a compressible finite-difference code in contrast to the spectral one used above), so the amplification effect appears to be numerically robust. We note that there have been earlier indications of nonhelical turbulence amplifying large-scale magnetic field in the presence of a large-scale shear associated with mean flows in numerical experiments that used constant-in-time sinusoidal forcing functions [18, 19]. Another example of large-scale magnetic fields generated by a combination of nonhelical turbulence and a mean flow is the numerical experiments with Taylor-Green forcing 20]. One might speculate that the shear provided by the mean flow in such systems could act in a way qualitatively similar to a linear shear and give rise to mean-field amplification.

Another context in which the shear dynamo may be important is accretion disks, where turbulence is driven by the magnetorotational instability (MRI) [6]. The MRI requires a (weak) large-scale field and gives rise to velocity and magnetic fluctuations at small scales. This turbulence could then conceivably couple to the largescale Keplerian shear and amplify the large-scale field, thus closing the loop. While the feasibility of such an MRI-dynamo mechanism will be the object of a separate study, we have verified already that the shear dynamo continues to work in the presence of rotation.

We thank A. Brandenburg, L. Kitchatinov, G. Lesur, G. Ogilvie, J. Papaloizou, D. Shapovalov, D. Sokoloff and especially F. Rincon for valuable discussions. Simulations were done on UKAFF, NCSA (Illinois) and Cambridge HPCF. This work was supported by STFC (T.H., A.A.S), Newton Trust (T.A.Y, T.H.), US DOE CMPD (A.B.I.), the Royal Society (I.R., A.B.I.) and the Leverhulme Trust Network for Magnetized Plasma Turbulence.

[1] G. K. Batchelor, Proc. R. Soc. London A 201, 405 (1950); A. P. Kazantsev, Sov. Phys.-JETP 26, 1031 (1968); Ya. B. Zeldovich et al., J. Fluid Mech. 144, 1 (1984); M. Meneguzzi, U. Frisch, and A. Pouquet, Phys. Rev. Lett. 47, 1060 (1981).

[2] A. A. Schekochihin et al., Astrophys. J. 612, 276 (2004).

[3] A. A. Schekochihin et al., New J. Phys. 9, 300 (2007).

[4] K.-H. Rädler and M. Rheinhardt, Geophys. Astrophys. Fluid Dyn. 101, 117 (2007).

[5] M. Ossendrijver, Astron. Astrophys. Rev. 11, 287 (2003).

[6] S. A. Balbus and J. F. Hawley, Rev. Mod. Phys. 70, 1 (1998); J.-F. Donati et al., Nature 438, 466 (2005).

[7] K. T. Chyzy et al., Astron. Astrophys. 355, 128 (2000); B. M. Gaensler et al., Science 307, 1610 (2005).

[8] R. Monchaux et al., Phys. Rev. Lett. 98, 044502 (2007).

[9] I. Rogachevskii and N. Kleeorin, Phys. Rev. E 68, 036301 (2003); Phys. Rev. E 70, 046310 (2004).

[10] E. T. Vishniac and A. Brandenburg, Astrophys. J. 475, 263 (1997); N. A. Silant'ev, Astron. Astrophys. 364, 339 (2000); M. R. E. Proctor, Mon. Not. R. Astron. Soc. 382, L39 (2007); N. Kleeorin and I. Rogachevskii, Phys. Rev. E 77, 036307 (2008).

[11] E. G. Blackman, Astrophys. J. 496, L17 (1998).

[12] V. Urpin, Mon. Not. R. Astron. Soc. 308, 741 (1999); Phys. Rev. E 65, 026301 (2002).

[13] K.-H. Rädler and R. Stepanov, Phys. Rev. E 73, 056311 (2006); G. Rüdiger and L. L. Kitchatiov, Astron. Nachr. 327, 298 (2006).

[14] A. Brandenburg, Astrophys. J. 550, 824 (2001).

[15] E. N. Parker, Astrophys. J. 122, 293 (1955).

[16] G. I. Ogilvie, unpublished (1998); Y. Lithwick, Astrophys. J. 670, 789 (2007).

[17] T. Elperin, N. Kleeorin, and I. Rogachevskii, Phys. Rev. E 68, 016311 (2003).

[18] A. Brandenburg, Astrophys. J. 625, 539 (2005).

[19] D. Shapovalov, private communication (2006).

[20] Y. Ponty et al., Phys. Rev. Lett. 94, 164502 (2005); P. D. Mininni et al., Astrophys. J. 626, 853 (2005).

[21] We found that the shear dynamo effect disappears when Re and Rm are too small, as predicted by SOCA [13]. 